\title{
The Design and Implementation of Intelligent Transportation Web Services
}

\author{
Chun-Hsin $\mathrm{Wu}^{1}$, Da-Chun $\mathrm{Su}^{1}$, Justin Chang ${ }^{1}$, Chia-Chen Wei ${ }^{1}$, Kwei-Jay Lin ${ }^{2}$, and Jan-Ming Ho ${ }^{1}$ \\ ${ }^{1}$ Instidute of Information Science \\ Academia Sinica, Nankang \\ Taipei, Taiwan 115 \\ \{wuch, starking, justin, ccwei, hoho\} \\ @iis.sinica.edu.tw \\ ${ }^{2}$ Dept. of Electrical Engineering and Computer \\ Science \\ University of California, Irvine \\ Irvine, CA, USA 92697 \\ klin@ece.uci.edu
}

\begin{abstract}
This paper presents the design and implementation of an intelligent transportation web service (ITWS) and studies various QoS requirements for the web service infrastructure. In particular, we pay attention to the performance issues on ITWS data transmission and compression. Since SOAP message is a textual document, we can improve the data transmission time by compressing data on transmission. In our experiment, compressing data before or after SOAP serialization both can reduce the size of transmitted message down to as little as $2 \%$ and cut the response time to a quarter of the original message.
\end{abstract}

\section{Introduction}

In the competitive and fast changing environment, trading, marketing, and advertising products and services using the Internet has been a very essential and significant part of a company's strategic planning [1,2]. To fulfill the needs of customers and enterprises, e-commerce user interface should be dynamic, interoperable and should have fast response time while being extremely efficient.

The emergence of web service technology targets to accomplish the above mentioned characteristics by allowing software components as services to be reused and integrated over the Internet $[3,4,5]$. It is language and platform independent and can easily pass through firewall.

Intelligent Transportation System (ITS) research aims to apply advanced information processing, management, and communication technologies to improve the performance of public transportation system [6]. In the past few years, the integration of ITS and e-commerce has been strongly promoted by researchers from government agencies and academics. The popularity of cheap and portable digital devices, the ubiquitous availability of wireless Internet, and the ever-expanding public roadway traffic make the application of web services to ITS a promising solution to our traffic problems.

This paper presents an implementation of an intelligent transportation web service (ITWS) and analyzes various web service QoS requirements for web service infrastructure. In contrast to common business applications, ITWS is designed to be a web service infrastructure, to be used as a modular component by other enterprises or web service providers for application development. While achieving the goal of increasing the reusability of modular applications, it also decreases the cost for service implementation. Figure 1 shows some potential applications on ITWS, including traffic information services, logistic service providers, tour and travel services, medical and emergency managements, etc. Therefore a powerful yet flexible ITWS will have a very significant impact to the society and make great contributions to human life in general.

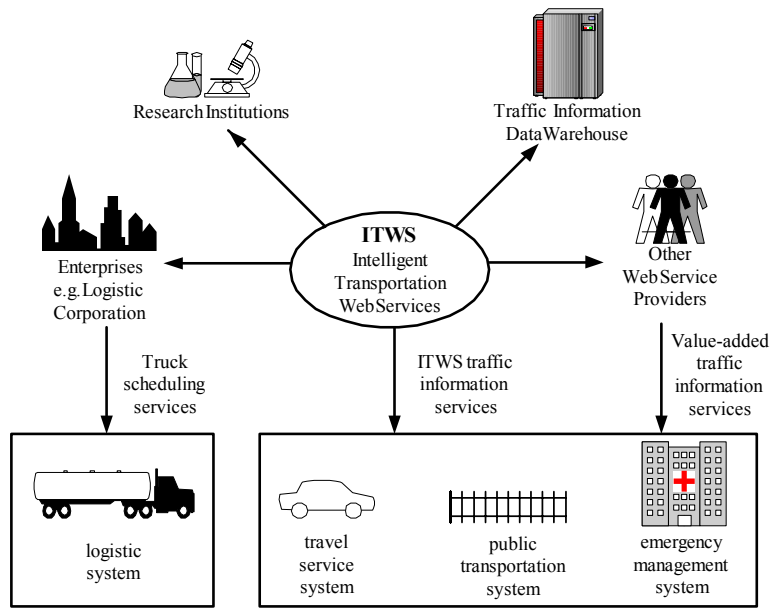

Figure 1. ITWS applications 
The remaining sections are organized as follows. Section 2 introduces the ITWS architecture. Section 3 discusses performance issues for QoS Web services, and section 4 concludes the paper.

\section{ITWS System}

The organization of the proposed ITWS system is shown in Figure 2. The Taiwan Area National Freeway Bureau (TANFB) constantly collects vehicle speed information from loop detectors installed on the highways, and provides the real-time raw traffic data in proprietary formats through the TANFB website [7]. So we develop an ITWS crawler that periodically fetches and parses the raw traffic data from the TANFB website, and then stores the interesting information into the local ITWS database. The ITWS server retrieves the local ITWS database to serve web service requests.

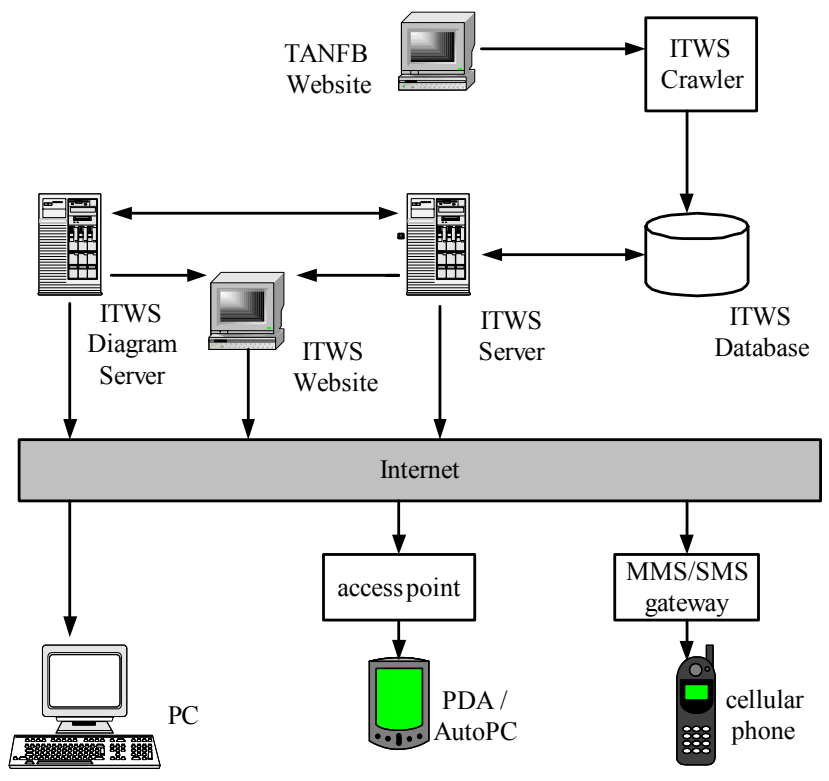

Figure 2. ITWS system architecture

In order to provide more user-friendly interfaces, we develop an ITWS diagram server to generate graphical traffic visualizations using the traffic data from the ITWS Server via the SOAP protocol. These traffic visualizations can be generated on-line, or off-line and pre-stored on the server. An ITWS web site is also created to provide the main human interface to ITWS. Based on the web service technology and traffic data from ITWS, external web service providers can customize the raw traffic data into various kinds of services with different data inspection and analysis processes. Furthermore, end users can access ITWS services through different types of devices (e.g. PDA, cellular phone, etc.). In addition, with the simplicity to incorporate ITWS modular applications with RPC calls, web service developers can further enhance the reusability and distributed characteristic. Also, the ITWS system further facilitates ITS researches for academic or government agencies.

\section{Performance Issues in ITWS}

The XML-based SOAP provides a seamless remote procedure call (RPC) mechanism between service providers in either synchronous or asynchronous way. In the synchronous mode, one who invokes a function provided in the web service is blocked until the result is returned. A long response time may be experienced if the request is made to a heavily loaded web server. Given the fast proliferation of web services, only service providers that offer quality services will eventually prosper $[8,9]$. In this section, we discuss the QoS issues in Web Service, and examine the performance and reliability of our system.

QoS predominantly contributes to the success and popularity of a web service [10] and includes several properties: including Availability, Accessibility, Security, Reliability, and Performance. Availability is the percentage of time that a service is operating. Accessibility is the percentage of requests that are successfully served by the services. Security aspects includes confidentiality of the data transferred, non-repudiation of request or message and resilience of denial-of-service attacks. Performance, often measured in throughput or response time, is the rate a service processes request or the time taken between invocation of a request and reception of a response respectively. Reliability is the percentage of requests that fails during a time period.

\subsection{QoS support in ITWS}

For intelligent transportation systems, QoS parameters such as accessibility, performance and reliability are considered to be especially important. We discuss these properties as follows.

Accessibility. In our implementation, the ITWS servers that provide services to clients can distinguish different requester's performance needs and responds with the most effective service in terms of both time and cost. Moreover, the service generates different responses to the ITWS web application interface or cellular phone display. Hence, ITWS provides a scalable device-adaptive design.

Performance. Web services for sharing information such as weather forecasting services, stockbroker services or traffic information services often require the transmission of massive amount of data. Therefore, data transmission time is critical to system performance. If not 
properly managed, detrimental degradation can be imposed to the performance of the entire system. The standardized way for web service information exchange, the SOAP protocol, is based on the XML syntax which contains bulky tags and is stored in plain text format. Several studies have tried to solve the problems of busy and heavy loaded web service traffic. One solution suggested is to compress/decompress the SOAP message right before and after data transmission. We will discuss this issue later.

Reliability. In this project, data reliability is enforced in the data prediction stage in terms of the validity of a historical data. Typically, the data reconstruction ratio is approximately $15 \%$, while sensor failure contributes to the majority of the total errors. Any ratio deviates greatly from the expected value will be discarded, and then proceeds to the next entry for consideration. For example, in Table 1, data entry on Oct 30, 2002 is uncommonly large and is rejected on data selection. In this way, we can reduce the chance of reliability degradation due to the use of unreliable data.

Table 1: Lost Data Reconstruction Ratio

\begin{tabular}{|c|c|c|c|}
\hline \multirow{2}{*}{$\begin{array}{c}\text { Date } \\
(2002)\end{array}$} & \multicolumn{2}{|c|}{ Source of Error } & $\begin{array}{c}\text { Total } \\
\text { Reconstruction } \\
\text { Ratio (\%) }\end{array}$ \\
\cline { 2 - 3 } & Sensor (\%) & Other Causes (\%) & 14.33 \\
\hline $10 / 24$ & 11.62 & 2.70 & 16.22 \\
\hline $10 / 25$ & 14.76 & 1.45 & 14.41 \\
\hline $10 / 26$ & 12.74 & 1.66 & 13.15 \\
\hline $10 / 27$ & 11.27 & 1.87 & 15.45 \\
\hline $10 / 28$ & 11.49 & 3.95 & 12.54 \\
\hline $10 / 29$ & 10.45 & 2.80 & 50.91 \\
\hline $10 / 30$ & 45.91 & 5.00 & \\
\hline
\end{tabular}

\subsection{SOAP Message Compression}

To improve the performance or to enhance the security of web service, we have implemented compression functions to handle SOAP messages. In our implementation, we override the SOAP Extension class provided by the Microsoft .NET framework to manipulate SOAP requests and responses at the SOAP message processing stage. In Figure 3, we show the SOAP message transitions between a web service consumer and provider for SOAP serialization approaches. When a web service consumer invokes a web service request, the proxy class puts the data into a SOAP message format and the message is then sent over the network. After a web service provider receives the SOAP message, it reconverts the SOAP message back to its original data types and processes it according to the requested web service method.

In Figure 3(a), when the processed data is ready, the web service provider converts the requested data into another SOAP message format. But instead of sending it directly to the consumer, the formatted SOAP message is compressed first at the AfterSerialize stage of SOAP Processing and then sent back to the web service consumer. Upon receiving the reply message by the web service consumer, the compressed SOAP message is decompressed at the BeforeDeserialize stage and then reformatted back to the original data types and returns the response to the calling function.

The conversions from the original data types to SOAP format and vice versa are referred to as serialization and deserialization in the .NET jargon. Also noted that, since Web service is still not fully standardized, SOAP message header used for web service client/server communication may still evolve in the near future. So we only compress the content of the body but not the header to warrant the service can still function properly after data compression. In this way, our message compression implementation can still be used for new versions of SOAP data format to be adopted in the future.

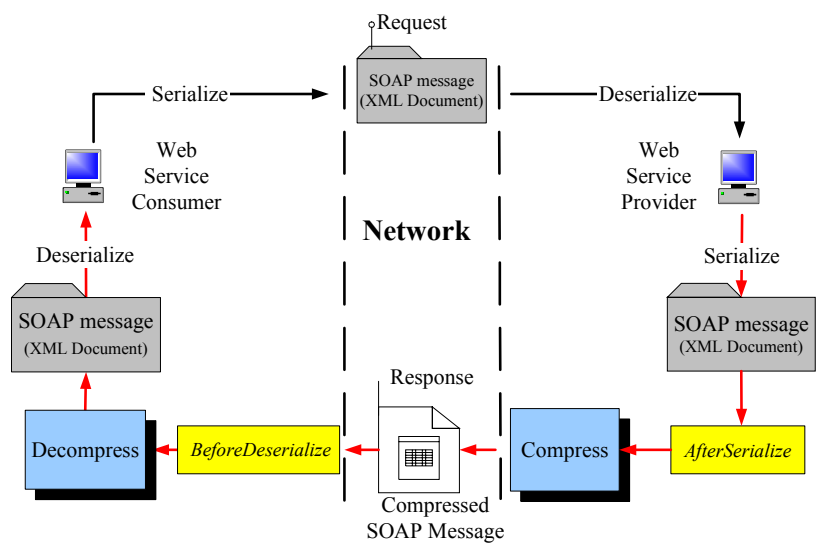

(a). Compression after SOAP serialization

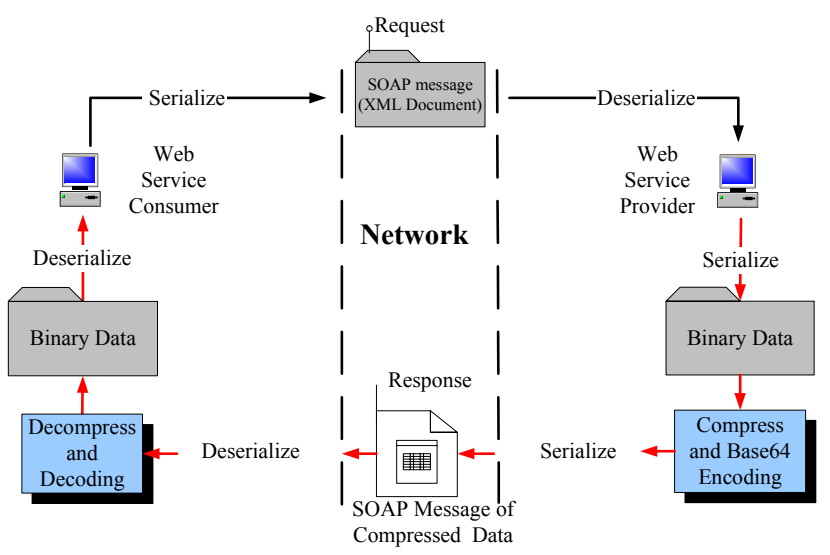

(b). Compression before SOAP serialization

Figure 3. SOAP message processing 
We also examine the efficiency of an alternative approach as shown in Figure 3(b). In this binary serialization approach, the data is first serialized into binary stream, and then compressed before the SOAP serialization stage.

Intuitively, these approaches can considerably reduce the data size and round-trip time latency. We have conducted experiments on a Pentium-4 PC with $1.7 \mathrm{GHz}$ microprocessor and 512MB RAM for two important web methods: N1_day_traffic and OD_Estimation. Table 2 shows the compression ratios for both approaches and Table 3 shows their round-trip time latencies. These results show that the compression of huge SOAP messages delivered between web service providers and consumers is very effective to improve system performance for ITWS either with SOAP or binary serialization. The binary serialization approach generally performs better in both compression ratio and latencies. However, the SOAP serialization approach is directly supported by Web Services standard, but extra works of understanding the implementation details of the binary serialization approach for web service users are needed.

Table 2. Efficiencies of message compression

(a). Compression after SOAP serialization

\begin{tabular}{|l|c|r|r|}
\hline \multirow{2}{*}{ Web Method } & \multicolumn{2}{|c|}{ Data Size (bytes) } & \multirow{2}{*}{$\begin{array}{c}\text { Compression } \\
\text { Ratio (\%) }\end{array}$} \\
\cline { 2 - 3 } & \multicolumn{1}{|c|}{ Original } & Compressed & \multicolumn{1}{c|}{98} \\
\hline N1_day_traffic & $8,172,400$ & 105,542 & 97 \\
\hline OD_Estimation & $44,218,584$ & $1,121,116$ & \\
\hline
\end{tabular}

(b). Compression before SOAP serialization

\begin{tabular}{|l|r|r|r|}
\hline \multirow{2}{*}{ Web Method } & \multicolumn{2}{|c|}{ Data Size (bytes) } & \multirow{2}{*}{$\begin{array}{c}\text { Compression } \\
\text { Ratio (\%) }\end{array}$} \\
\cline { 2 - 3 } & Original & Compressed & \multicolumn{1}{c|}{93.47} \\
\hline N1_day_traffic & 744,922 & 48,616 & 69.90 \\
\hline OD_Estimation & $11,270,704$ & $3,392,260$ & \\
\hline
\end{tabular}

Table 3. Round-trip time latencies of message compression

(a). Compression after SOAP serialization

\begin{tabular}{|l|c|l|c|c|}
\hline \multirow{2}{*}{ Web Method } & \multicolumn{4}{|c|}{ Average Time (sec.) } \\
\cline { 2 - 5 } & Compression & Transfer & Decompression & Round Trip \\
\hline N1_day_traffic & 0 & 15.834 & 0 & 16.143 \\
\hline $\begin{array}{l}\text { N1_day_traffic } \\
\text { with compression }\end{array}$ & 2.627 & 0.203 & 0.037 & 3.615 \\
\hline
\end{tabular}

(b). Compression before SOAP serialization

\begin{tabular}{|l|c|c|c|c|}
\hline \multirow{2}{*}{ Web Method } & \multicolumn{4}{|c|}{ Average Time (sec.) } \\
\cline { 2 - 5 } & Compression & Transfer & Decompression & Round Trip \\
\hline N1_day_traffic & 0 & 0.74 & 0 & 3.073 \\
\hline $\begin{array}{l}\text { N1_day_traffic } \\
\text { with compression }\end{array}$ & 0.1027 & 0.1255 & 0.0625 & 2.596 \\
\hline
\end{tabular}

\section{Conclusion}

In this paper we examine the feasibility of applying web services technology to intelligent transportation systems. We develop an Intelligent Transportation Web Service (ITWS) that can be utilized by different web service providers, clients, or enterprises on request. We also examine the performance issues on data transmission and compression for ITWS. Two approaches that compress data before or after SOAP serialization are evaluated. Both the compression approaches can reduce message size and transmission latency significantly, but there is a trade-off among development effort and compression performance. In our experiences, web services technology did reduce the complexity of system integration, but the performance degradation issues need be addressed.

\section{References}

[1] Aoyama, M., "A business-driven Web service creation methodology," in the Proceedings of Symposium on Applications and the Internet (SAINT) Workshops, 2002 Page(s): 225-228

[2] M. Aoyama, S. Weerawarana, H. Maruyama, C. Szyperski, K. Sullivan, and D. Lea, "Web services engineering: promises and challenges," in the Proceedings of the $24^{\text {th }}$ International Conference on Software Engineering, 2002, Page(s): 647-648

[3] M. Aoyama, "Intelligent Software Services over the Internet," E. Kawaguchi, et al. (eds.), Information Modeling and Knowledge Bases, IX, IOS Press, Feb. 2000, pp. 128-135

[4] P. Brereton, et al., The Future of Software, CACM, Vol. 42, No. 21, Dec. 1999, pp. 78-84.

[5] S. Aissi, P. Malu, K. Srinivasan, "E-business process modeling: the next big step," Computer, Volume: 35 Issue: 5 , May 2002 Page(s): 55-62

[6] J. Y. Kwon, B. Coifman, and P. Bickel, "Day-to-day travel time trends and travel time prediction from loop detector data," Transportation Research Record no. 1717, Transportation Research Board, 2000, pp 120-129

[7] Taiwan Area National Freeway Bureau website, http://www.freeway.gov.tw/link_e.htm

[8] H.S. Chiu, and C. Yang, "Beyond e-commerce software quality: Web services effectiveness," in the Proceedings of the 2001 Asia-Pacific Conference on Quality Software, pp $397-405$

[9] M. Litoiu, "Migrating to web services - latency and scalability," in the Proceedings of the Fourth International Workshop on Web Site Evolution, 2002, pp. 13-20

[10] D. A. Menasce, "QoS issues in web services," Internet Computing, IEEE, Vol. 6, No. 6, Nov/Dec 2002, pp. 72-75 